
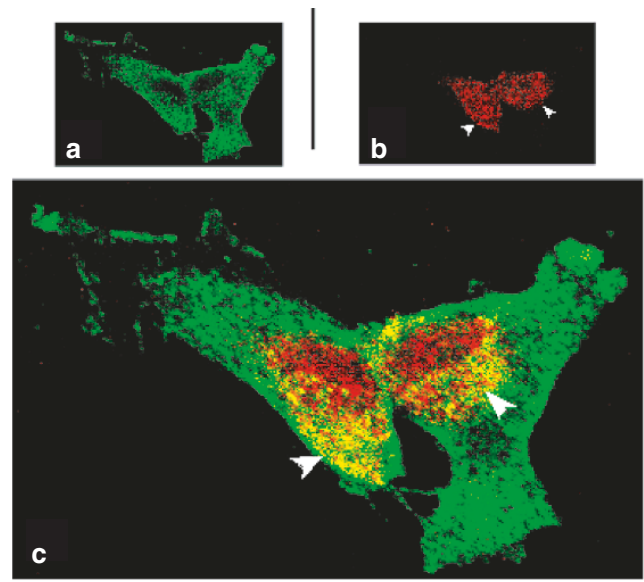
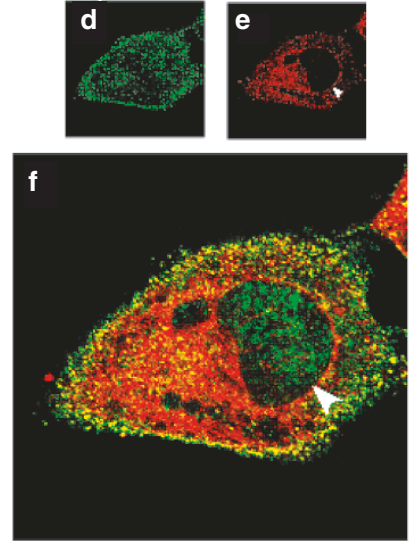
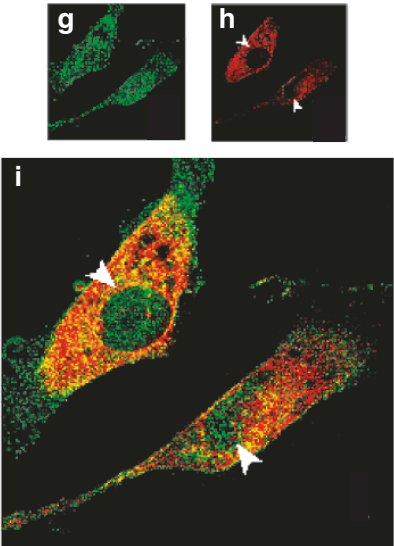

Figure 2. Localization of EGFR in the nucleus and other organelles in MDA-MB-468 cells. a, EGFR staining was performed using mouse anti-EGFR antibody (Ab-12, NeoMarkers) followed by FITC-labeled donkey anti-mouse IgG (Jackson ImmunoResearch). EGFR was found in the nucleus as well as in the cytoplasm. b. To confirm the nuclear localization of EGFR, the nuclei were stained using mouse anti-lamin A/C antibody (Santa Cruz Biotechnology) followed by FITC-labeled donkey anti-mouse IgG. EGFR staining using rabbit anti-EGFR antibody (sc03, Santa Cruz Biotechnology) followed by Texas-Red-labeled goat anti-rabbit IgG (Jackson ImmunoResearch). The overlay image of the two shows that EGFR signals are also inside the areas confined by lamin staining, i.e., the nuclei. c, ER staining using mouse anti-Calnexin antibody (BD Transduction Laboratories) followed by FITC-labeled donkey anti-mouse IgG. EGFR staining using rabbit anti-EGFR antibody followed by Texas-Red-labeled goat anti-rabbit IgG. d, ER staining using rabbit anti-Calregulin antibody (Santa Cruz Biotechnology) followed by Texas-Red-labeled goat anti-rabbit IgG. EGFR staining using mouse anti-EGFR antibody followed by FITC-labeled donkey anti-mouse IgG. Nuclear staining using DAPI further confirms the nuclear area defined by ER staining. $\mathbf{a}$ and $\mathbf{b}$ show nuclear localization of EGFR; and $\mathbf{c}$ and $\mathbf{d}$ clearly demonstrate that most of nuclear EGFR staining is not co-localized with ER staining. Similar results were obtained when another cell line, A431 was used for staining (data not shown).

EGFR can bind directly to the promoter region of cyclin D1 in vivo (Fig. 7b in the paper). The protein localized in ER membrane will not associate with chromatin; therefore, direct binding of EGFR to a promoter in vivo is rock-solid data to support the nuclear localizaton of EGFR. As to the confocal results shown in Fig. 1d (ref. 4), the picture might be too small to have good resolution. The original figure has much better resolution. But all the biochemical evidence (Figs $2-7$ in ref. 4 ) and the data presented in this correspondence clearly supports the localization of EGFR in the nucleus.
1. Lin, S.-Y., et al. Nature Cell Biol. 3, 802-808 (2001). 2. Pelkmans, L., Kartenbeck, J, \& Helenius, A. Nature Cell Biol. 3, 473-483 (2001)

3. Reilly, J. F. \& Maher, P. A. J. Cell Biol. 152, 1307-1312 (2001). 4. Peng, H. et al. Mol. Biol. Cell 12, 449-462 (2001).

5. Bargmann, C. I., Hung, M. C. \& Weinberg, R. A. Cell 45, 649-657 (1986)

Lilly Bourguignon*, Keng-hsueh Lan†, Patrick Singleton*, Shiaw-Yih Lin§, Dihua Yu†§, and Mien-Chie Hung†‡§

${ }^{*}$ Department of Medicine, University of California San Francisco and Endocrine Unit at the VA Medical Center, San Francisco, CA 94121, USA;
†Department of Surgical Oncology and ¥Department of Molecular and Cellular Oncology, University of Texas MD Anderson Cancer Center, Houston, TX 77030-4009, USA Se-mail:mhung@mdanderson.org

\section{Cells and gels}

To the editor - I read Michael Klymkowsky's recent review of my book, Cells, Gels and the Engines of Life (Nature Cell Biol. 3, E213; 2001), and find myself struggling to understand how his observations lead to his conclusions.

Klymkowsky admits that at least some of the challenges to current orthodoxy may be substantive. Yet, he dismisses such challenges as "anecdotal," notwithstanding some 450 cited references. He then goes on to declare that the "cross-checked" findings of many studies (none cited) leave little room for doubt that current views must be rock solid. Given such certainty, he is forced to conclude that questions such as those raised in the book do harm to science.

I was taught that scientific theories could never be proved; that no matter how much evidence could be marshalled in support of a theory, a new, conflicting observation has the potential to turn that theory on its ear. Klymkowsky has apparently learned from a different teacher. With a sweep of the hand, he dismisses the wealth of contradictory evidence presented in the book as "anecdotal," and prefers to bank on all of those ineffable "cross-checked" findings. Perhaps he could explain why findings that fit current views should be given more weight than those that do not fit.

The author also takes a jab at the presentation's "folksy" style. Deviating from the stuffy, jargon-filled style that typifies much scientific writing (and obscures flaws in reasoning) is regarded as unscientific.

Klymkowsky may be surprised to find that the material he dismisses as "muckmaking" is in growing demand worldwide-on the plenary agenda at international scientific venues, and increasingly in the classroom at major universities. Cells, Gels and the Engines of Life is becoming a scientific best-seller, with translations pending in three languages. The community is evidently hungry for a critical, no-holds barred, evaluation of entrenched paradigms, and for exposure to potentially more productive paradigms - even if the presentation style may border on the "folksy."

Gerald H. Pollack

Dept. of Bioengineering, University of Washington, Seattle, Washington 98195, USA e-mail:ghp@u.washington.edu 